\title{
Two-Dimensional Synthetic Aperture Radiometry over Land Surface During Soil Moisture Experiment in 2003 (SMEX03)
}

\author{
Dongryeol Ryu \\ Thomas J. Jackson \\ and Rajat Bindlish \\ USDA-ARS Hydrology and \\ Remote Sensing Laboratory \\ Beltsville, Maryland, USA \\ Email: Tom.Jackson@ARS.USDA.GOV
}

\author{
David M. Le Vine \\ and Michael Haken \\ Instrument Science Branch \\ NASA Goddard Space Flight Center \\ Greenbelt, Maryland, USA \\ Email: David.M.LeVine@NASA.GOV
}

\begin{abstract}
Microwave radiometry at low frequencies (L-band, $\sim 1.4 \mathrm{GHz}$ ) has been known as an optimal solution for remotesensing of soil moisture. However, the antenna size required to achieve an appropriate resolution from space has limited the development of spaceborne L-band radiometers. This problem can be addressed by interferometric technology called aperture synthesis. The Soil Moisture and Ocean Salinity (SMOS) mission will apply this technique to monitor global-scale surface parameters in the near future. The first airborne experiment using an aircraft prototype of this approach, the Two-Dimensional Synthetic Aperture Radiometer (2D-STAR), was performed in the Soil Moisture Experiment in 2003 (SMEX03). The L-band brightness temperature data acquired in Alabama by the 2DSTAR was compared with ground-based measurements of soil moisture and with C-band data collected by the Polarimetric Scanning Radiometer (PSR). Our results demonstrate a good response of the 2D-STAR brightness temperature to changes in surface wetness, both in agricultural and forest lands. The behavior of the horizontally polarized brightness temperature data with increasing view-angle over the forest area was noticeably different than over bare soil. The results from the comparison of 2D-STAR and PSR indicate a better response of the 2D-STAR to the surface wetness under both wet and dry conditions. Our results have important implications for the performance of the future SMOS mission.
\end{abstract}

\section{INTRODUCTION}

Soil moisture is an important land surface variable which affects the exchanges of energy and water between land and atmosphere. There have been a number of studies that have utilized airborne or spaceborne $\mathrm{C}$ - and $\mathrm{X}$-band microwave instruments in order to retrieve surface soil moisture over a large area [1]. Currently, the Advanced Microwave Scanning Radiometer (AMSR-E) provides global coverage of surface soil moisture that are retrieved primarily using $\mathrm{X}$-band data. However, L-band microwave (approximately $1.4 \mathrm{GHz}$ ) is known as the optimal choice for remote sensing of soil moisture and sea surface salinity due to its high sensitivities to the soil wetness and salinity of water [2], [3].A growing need for global-scale observation of soil moisture using Lband radiometer has motivated the Soil Moisture and Salinity
Mission (SMOS) by European Space Agency (ESA), which is scheduled to be launched in 2008 [3].

Conventional L-band remote sensing of soil moisture from space requires large antennas mechanically scanning in space in order to achieve a high spatial resolution over a large fieldof-view. This instrumental constraint on size can be overcome by using an interferometric technique called aperture synthesis. In aperture synthesis, microwave signals received by an array of small sensors are transformed to the brightness to achieve the spatial resolution of a single large antenna [4]. In addition to the advantages in size, the synthetic aperture radiometer can produce multi-angular brightness temperatures with a large field-of-view without scanning the instrument. SMOS is the first space mission which will deploy the interferometric technique for the remote sensing of soil moisture.

The first airborne experiment using a two-dimensional interferometer was carried out during the Soil Moisture Experiment in 2003 (SMEX03). The Two-Dimensional Synthetic Aperture Radiometer (2D-STAR) was flown onboard a NASA P3B aircraft from June to July, 2003 over four regional-scale sites (approximately $50 \mathrm{~km} \times 100 \mathrm{~km}$ ) located in Alabama, Georgia, and Oklahoma, USA. Land cover in the study areas includes various vegetation conditions such as bare soil, pasture, crop fields, and forest, and topographic conditions varying from flat or gently rolling plains to high-relief hilly area. During the experiment, 2D-STAR collected dual-polarized multi-angular brightness temperature data. The Polarimetric Scanning Radiometer (PSR) was mounted on the same aircraft adjacent to the 2D-STAR collecting C- and X-band data simultaneously with the 2D-STAR over the same regions. The concurrent operation of the 2D-STAR and PSR provided a unique opportunity to compare the features of the L-band synthetic aperture instrument with the $\mathrm{C}$ - and $\mathrm{X}$-band real aperture radiometer.

In this work, the general performance of the 2D-STAR over pasture, crops fields, and forest is presented and compared with the performance of PSR. Then, the implications of the results for the future SMOS mission and its potential advantages over 
currently operating spaceborne $\mathrm{C}-\mathrm{X}$-band instruments will be discussed.

\section{DATA SET DESCRIPTION}

A. Two-Dimensional Synthetic Aperture Radiometer (2DSTAR)

The 2D-STAR was developed under NASA's Instrument Incubator Program as a continuation of the previous onedimensional L-band interferometric radiometer, the Electronically Scanned Thinned Array Radiometer (ESTAR) [5]. The ESTAR successfully demonstrated the mapping capability of surface soil moisture at regional scales [2]. The limited flexibility of the antenna-array configuration in the 1-D array of the ESTAR has been greatly improved in the 2-D array of the 2DSTAR, and the single polarization mode and analog processing have evolved into dual polarization and digital processing [6].

Aperture synthesis is a relatively new technique in which an array of small antennas is utilized to achieve the resolution of a single large aperture antenna [4]. This interferometric technique was proposed to overcome the constraint on antenna size for spaceborne L-band radiometry [7]. Due to the large field of view of the small antennas, mechanical scanning is generally not required for interferometric radiometers. The 2DSTAR instrument is composed of a fully populated $11 \times 11$ array of small square antennas, each of which has dimensions of $6.86 \mathrm{~cm} \times 6.86 \mathrm{~cm}$. Each antenna is separated by 0.5 wavelength ( $\sim 10.6 \mathrm{~cm}$ at L-band) from adjacent antennas. Of the entire $11 \times 11$ elements, 21 antennas along the principle axes, which is a " + " shape configuration, were activated for operation and the tilt angle of the instrument was zero during SMEX03. Complex correlations of the signals from all the possible pairs of the activated 21 antennas are transformed to form a circular image of footprint. Each footprint is composed of numerous pixels, and the size and view-angle of the pixels increase as they are located farther from the center. In order to avoid distortion at larger view-angles, the radius of the circular footprint is limited to a cone with a vertex angle of 80 degrees, which is $\pm 40^{\circ}$ from nadir. The circular footprint was produced every 0.2 seconds during the experiment. The image processing resulted in a $10 \mathrm{~km}$ swath and an average resolution of $\sim 800 \mathrm{~m}$ at the nominal flight altitude of $7.7 \mathrm{~km}$ utilized in SMEX03.

\section{B. Soil Moisture Experiment in 2003 (SMEX03)}

One of the main objectives of SMEX03 was validating AMSR-E brightness temperature and soil temperature products using airborne and ground-based measurements collected over a wide range of land surface conditions [8]. SMEX03 was conducted over four regional-scale sites $(\sim 50 \mathrm{~km} \times 100 \mathrm{~km})$, which is equivalent to the size of 8 10 Equal-Area Scalable Earth Grid (EASE-Grid), located in Oklahoma (2 sites), Alabama (1 site), and Georgia (1 site) during the period of June and July in 2003. Among the four study sites, land-cover types and surface wetness conditions exhibited the largest ranges in Alabama during the SMEX03. In this work, airborne and ground-based data collected in Alabama will be presented.

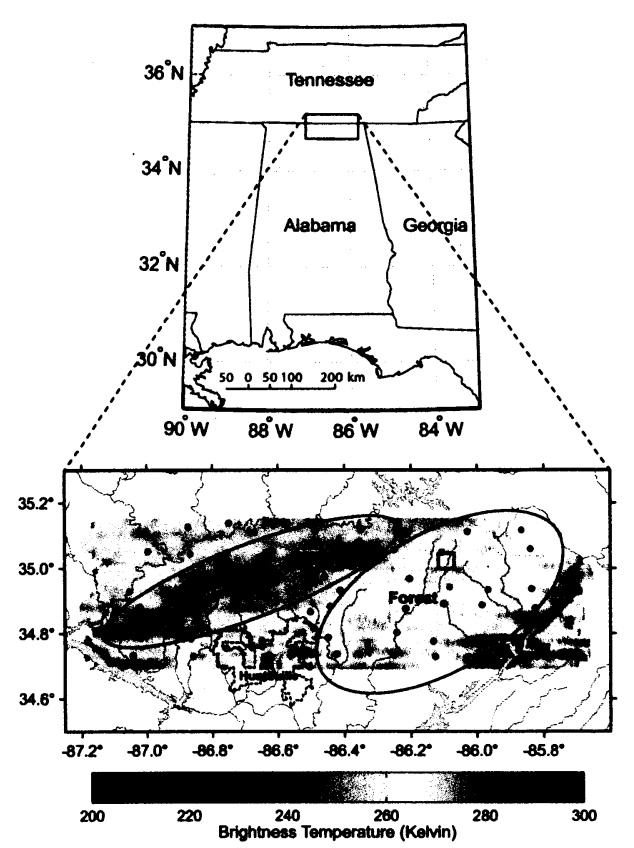

Fig. 1. (a) The location of Alabama study area. (b) The horizontally polarized brightness temerature data from 2D-STAR (June, 25, 2003) is superimposed with the rivers and lakes (light blue lines and polygons) in the study area. The ovals mark agricultural and forest lands in the study area, and red dots spread over the region are 58 ground-based sampling locations.

The Alabama study area is located near the AlabamaTennessee border and the Tennessee River Valley (Figure 1). The mapping area is oriented east-west, consisting of $2 \times 5$ EASE-Grid pixels. A horizontally polarized brightness temperature (TB-H) image collected by 2D-STAR on June 25 is superimposed with the rivers and lakes in Figure $1 \mathrm{~b}$. About $27 \%$ of the western part and $68 \%$ of the eastern part of the site is forested land, which matches the high TB-H area in the Figure 1b. The forested areas are characterized by significant relief and the dominant species are pines and oaks. Agricultural land spans from the southwestern to the northeastern portions of the region and accounts for about $40 \%$ of the site. Most of the agricultural land is composed of pastures and crops (corn, soybean, cotton, etc.) and it is characterized by flat or gently rolling topography.

During SMEX03, the NASA P-3B aircraft, equipped with 2D-STAR and PSR, collected data along four east-west flight lines on five days: June 25, 27, 29, 30, and July 2. Groundbased soil moisture measurements were taken concurrently with the airborne instruments at 58 sampling locations spread over the study area (purple dots in Figure 1b). The soil moisture in the top $5 \mathrm{~cm}$ was measured at three different points in each sampling location and averaged to represent the soil moisture content of the location. There were two major rainfall events over the study area during the sampling period: one between June 29 and June 30; and the other between June 30 and July 2, which provided moisture over most of the study area. 


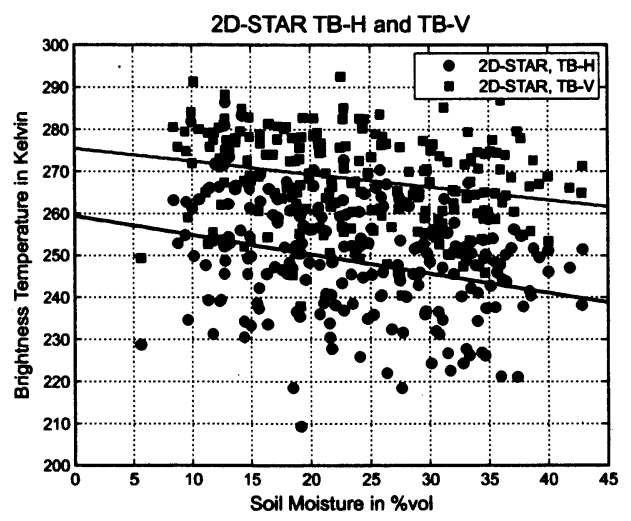

Fig. 2. Comparison of 2D-STAR TB-H/TB-V with ground-based measurements of surface soil moisture during SMEX03.

\section{Data Analysis}

The brightness temperature data product from the 2D-STAR is a time sequence of circular TB images. Each circular footprint contains numerous pixels, whose size and view-angle increase as the pixels are located farther from the center. Maximum view-angle of the footprint was limited to $40^{\circ}$ from nadir in order to avoid the distortion of images at large viewangles. With the instrument's integration time of 0.2 seconds, each footprint is separated from the adjacent ones by $\sim 50$ meters. Since the brightness temperature of a target changes with view-angle, we used 2D-STAR data with view-angles ranging from $30^{\circ}$ to $40^{\circ}$ to facilitate comparisons. The study area was not entirely covered by the TB data with low viewangles (less than $20^{\circ}$ ). The 2D-STAR TB data with the selected range of view-angles were mapped on the same grid as the PSR at a resolution of $800 \mathrm{~m}$.

The ground-based measurements of soil moisture at 58 locations were compared with the 2D-STAR pixels containing the sampling locations (Figure 2). In order to investigate the relationship between TB and view-angle in the agricultural and forest lands, TB-H and TB-V were sampled on June 25 for two rectangular areas (marked as red boxes in Figure 1b), which represented pasture/crop fields and forest. The viewangle varied from $0^{\circ}$ to $40^{\circ}$ (Figure 3). The entire images of TB from 2D-STAR and PSR were compared on a dry (June 25) and a wet (June 30) day to compare the ranges of the brightness temperature data from the instruments. These results are summarized in Figure 4.

\section{RESULTS AND DISCUSSION}

In this section, results from the comparison of the 2D-STAR data with the ground-truth data and TB-H/TB-V from the PSR are presented. Figure 2 shows the ground-based measurements of soil moisture versus horizontally and vertically polarized brightness temperature from 2D-STAR. The 800-m scale pixels containing the ground sampling locations were used for this comparison. Both TB-H and TB-V decreases with soil moisture linearly. Overall, TB-V is higher than TB$\mathrm{H}$ by $\sim 20 \mathrm{~K}$ and this difference in $\mathrm{TB}$ slightly increases
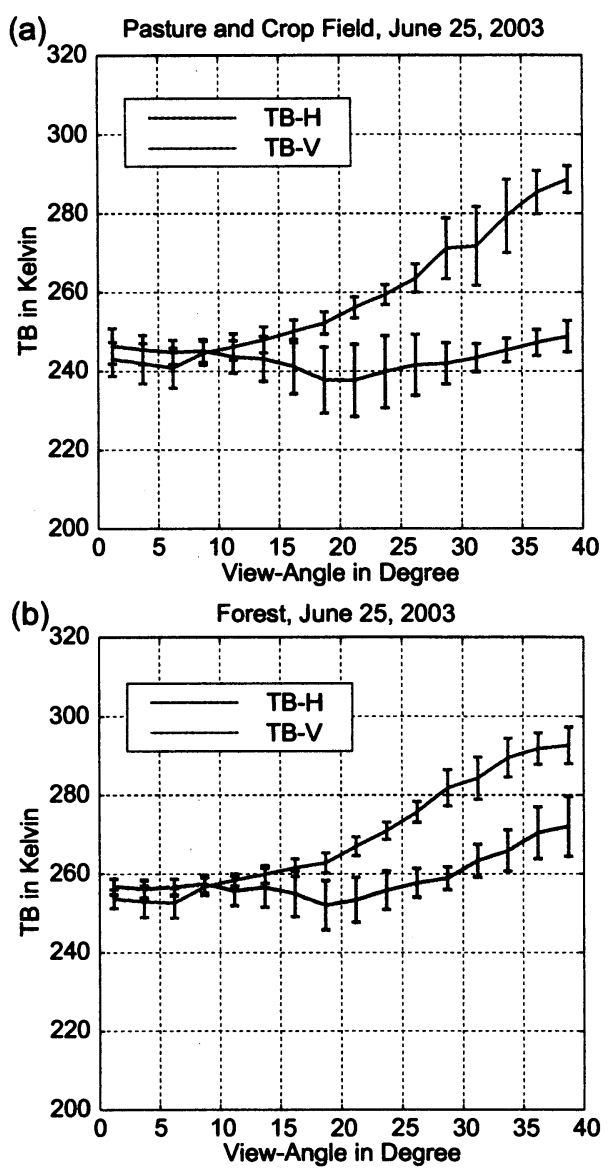

Fig. 3. The 2D-STAR brightness temperature (TB-H/TB-V) versus viewanlge observed in a) agricultural land (red box on the left in Figure lb) and b) forest (red box on the right in Figure lb) during SMEX03

with soil moisture. The correlation coefficient and residual error from the fit line were -0.264 and $14.5 \mathrm{~K}$ for TB-H, and -0.259 and $9.9 \mathrm{~K}$ for TB-V. The greater residual error of TB-H is related to the larger dynamic range of TB-H. Theoretically, the emissivity (brightness temperature divided by the true temperature of the target) of the land surface should display a strong negative correlation with the moisture content of the soil, however, the soil temperature data was not available at the time of this analysis. Variability of soil temperature for constant moisture content is likely a factor contributing to the weak correlation and large residual error of the brightness temperature. Another potential contributor to the observed weak correlation is the spatial variability of soil moisture. We used ground-truth data available at only one sampling location in each 800-m scale pixel of the 2D-STAR. Soil moisture spatial variability is about $6 \sim 8 \%$ vol at 800 $\mathrm{m}$ scale. However, the brightness temperature data from 2DSTAR still showed fairly good correlation with surface soil moisture, particularly in the forest areas.

One of the advantages of utilizing aperture synthesis is that the brightness temperature from multiple view-angle can be obtained, which can be useful in reducing the number 
of ancillary data required for retrieving soil moisture [9]. The brightness temperature of bare soil increases with viewangle for TB-V and decreases for TB-H [10]. This increasing or decreasing trend can be reduced when the radiometric signal is emitted from the rough surface. Figure 3 shows the brightness temperature versus view-angle of 2D-STAR sampled in pasture/crop field (Figure 3a) and forest (Figure 3b). The sampling areas are displayed in the Figure $1 \mathrm{~b}$ as red boxes. TB-V increased with view-angle in both sampling areas, whereas TB-H showed a rather flat pattern in the pasture/crop field and a slightly increasing pattern in the forest area. The observed trend of TB-H versus view-angle looks quite different from the previously reported patterns for bare soil, however, [11] reported increasing TB-H with view-angle when they included the diffus albedo from the land surface and vegetation in the radiative transfer model. The observed trend of TB-H requires further research because the behavior of TB$\mathrm{H}$ with view-angle is very important information in retrieving soil moisture from SMOS.

Figure 4 compares the brightness temperature from the 2DSTAR and PSR collected on a dry (June 25) and a wet (June 30) day during SMEX03. The dynamic ranges of 2D-STAR TB-H and TB-V are much wider than PSR and the difference increased as the field became wetter. The horizontally polarized brightness temperature showed better correlation than TB$\mathrm{V}$, and the correlation increased on the wet days, which was likely caused by the shallower sensing depth of PSR than the 2D-STAR.

\section{CONCLUSION}

The results from the first airborne experiment utilizing a two-dimensional synthetic aperture radiometer are presented. Although there was a considerable gap between the support scales of the 2D-STAR TB and ground-truth data, the brightness temperature data showed a fairly good (negative) correlation with ground-based measurements of surface soil moisture in both agricultural and forest lands. While TB-V from the 2D-STAR showed an increasing trend with viewangle as predicted by the theory, TB-H was rather flat or slightly increased with view-angle. This is likely caused by the diffuse scattering of rough surfaces and vegetation. The results from the comparison of the 2D-STAR and PSR indicate that 2D-STAR has a better response to the surface wetness under both wet and dry conditions. Our results have important implications for the potential advantages of the future SMOS mission over the currently operating spaceborne C-/X-band radiometers, particularly over the densely vegetated area.

\section{REFERENCES}

[1] E. G. Njoku, T. J. Jackson, V. Lakshmi, T. K. Chan, and S. V. Nghiem, "Soil moisture retrieval from AMSR-E," IEEE Trans. Geosc. Rem. Sens., vol. 41, no. 2, pp. 215-229, 2003.

[2] T. J. Jackson, D. M. Le Vine, A. Y. Hsu, A. Oldak, P. J. Starks, C. T. Swift, J. D. Isham, and M. Haken , "Soil moisture mapping at regional scales using microwave radiometry: The Southern Great Plains Hydrology Experiment, IEEE Trans. Geosc. Rem. Sens., vol. 37, no. 5, pp. 2136-2151, 1999. (a) 2D-STAR vs. PSR in Alabama on June 25, 2003

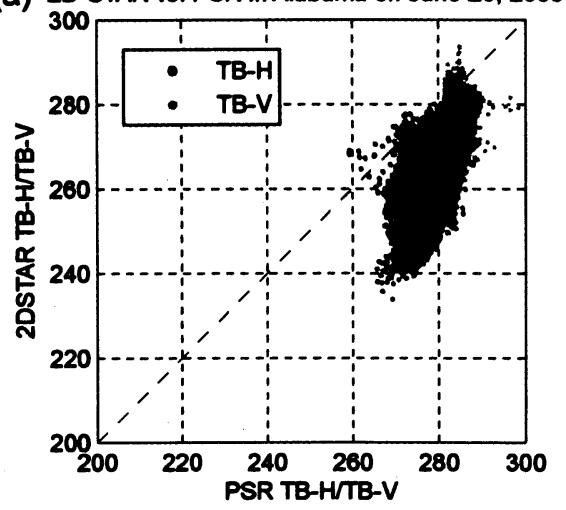

(b) 2D-STAR vs. PSR in Alabama on June 30, 2003

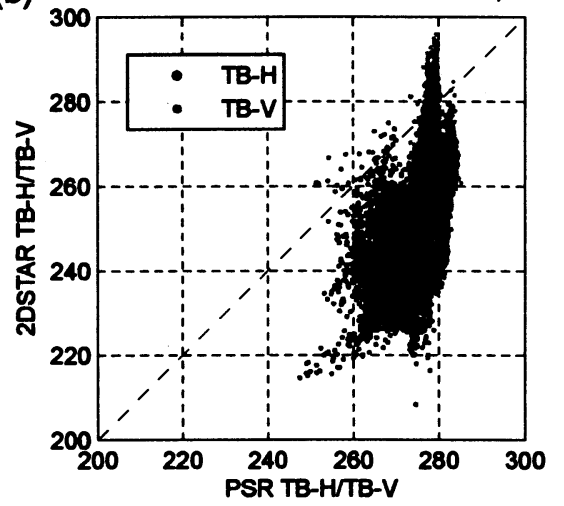

Fig. 4. Comparison of brightness temperature from 2D-STAR and PSR obtained on a) June 25 and b) June 30 during SMEX03

[3] Y. H. Kerr, P. Waldteufel, J. Wigneron, J. Martinuzzi, J. Font, and M. Berger, "Soil moisture retrieval from space: the soil moisture and ocean salinity mission (SMOS)," IEEE Trans. Geosci. Remote Sens., vol. 39, no. 8, pp. 1729-1735, 2001.

[4] N. Skou, and D. Le Vine, Microwave Radiometer Systems: Design and Analysis. Norwood, MA: Artech House, 2006.

[5] D. M. Le Vine, T. J. Jackson, C. T. Swift, M. Haken, and S. Bidwell, "ESTAR measurements during the Southern Great Plains experiment (SGP99)," IEEE Trans. Geosc. Rem. Sens., vol. 39, no. 8, pp. 16801685, 2001.

[6] D. M. Le Vine, T. J. Jackson, and M. Haken, "Initial images of the Synthetic Aperture Radiomter 2D-STAR," IEEE Trans. Geosc. Rem. Sens., to be published.

[7] C. S. Ruf, C. T. Swift, A. B. Tanner, and D. M. Le Vine, "Interferometric synthetic aperture microwave radiometry for the remote sensing of the earth," IEEE Trans. Geosc. Rem. Sens., vol. 26, no. 5, pp. 597-611, 1988.

[8] T. J. Jackson, R. Bindlish, A. J. Gasiewski, B. Stankov, M. Klein, E. G. Njoku, D. Bosch, T. L. Coleman, C. A. Laymon, and P. Starks, "Polarimetric scanning radiometer $\mathrm{C}$ - and X-band microwave observations during SMEX03," IEEE Trans. Geosc. Rem. Sens., vol. 43, no. 11, pp. 2418-2430, 2005.

[9] J. P. Wigneron, P. Waldteufel, A. Chanzy, J. C. Calvet, and Y. Kerr, "Two dimensional microwave interferometer retrieval capabilities over land surfaces (SMOS mission)," Remote Sens. Environ., vol. 73, pp. 270-282, 2000.

[10] F. T. Ulaby, R. K. Moore, and A. K. Fung, Microwave Remote Sensing: Active and Passive - Volume III: From Theory to Applications. Norwood, MA: Artech House, 1986.

[11] R. H. Lang, N. Chauhan, C. Utku, and D. M. Le Vine, "L-band Active and Passive Sensing of soil moisture through forests," IEEE MicroRad, pp. 193-196, 2006. 